Archive for

Organic Chemistry

Arkivoc 2020, part vii, 201-213

\title{
Electrochemically induced tandem Knoevenagel-Michael assembling of aldehydes with kojic acid: direct and efficient arylbis[3-hydroxy-6- (hydroxymethyl)-4-oxo-4H-pyran-2-yl]methanes formation
}

\author{
Michail N. Elinson, ${ }^{* a}$ Yuliya E. Ryzhkova, ${ }^{a}$ Anatoly N. Vereshchagin, ${ }^{a}$ Natalia A. Leonova, ${ }^{a}$ Alexandra P.
} Minaeva, ${ }^{a, b}$ and Mikhail P. Egorov ${ }^{a}$

${ }^{a}$ N. D. Zelinsky Institute of Organic Chemistry, Leninsky prospect 47, 119991 Moscow, Russia

${ }^{b}$ D. I. Mendeleev University of Chemical Technology of Russia, Miusskaya square 9, 125047 Moscow, Russia

Email: elinson@ioc.ac.ru

Dedicated to Prof. Jan Bergman on the occasion of his $80^{\text {th }}$ anniversary

Received 04-11-2020

Accepted 08-26-2020

Published on line 09-06-2020

\section{Abstract}

The electrochemically induced tandem reaction of aldehydes and two equivalents of kojic acid has been carried out in alcohols in an undivided cell in the presence of sodium halides. It led to the selective formation of substituted arylbis[3-hydroxy-6-(hydroxymethyl)-4-oxo-4H-pyran-2-yl]methanes in 71-98\% yields and with 240-330\% current efficiency. This new electrocatalytic process provides green, useful and efficient way to two kojic acid fragments separated by C-aryl-substituted spacer, which are promising compounds for different biomedical applications.
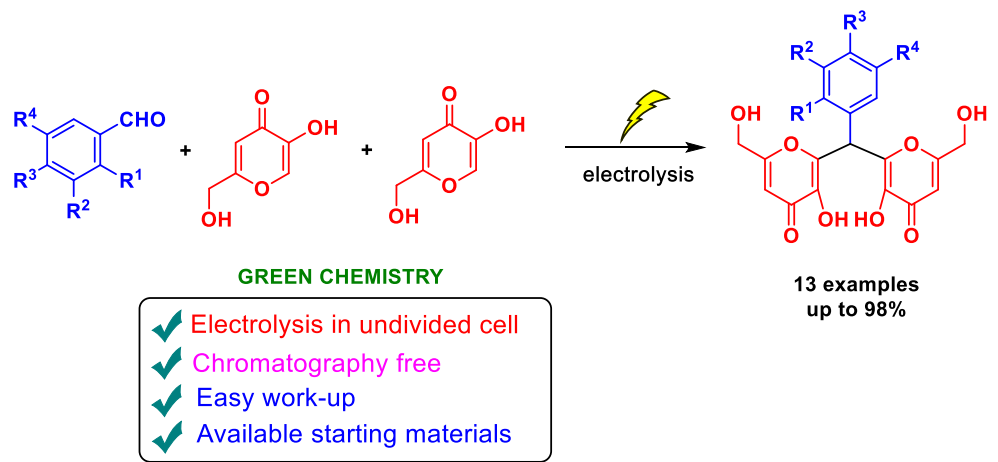

13 examples up to $98 \%$

Keywords: Electrocatalysis, tandem reaction, aldehydes, kojic acid, arylbis[3-hydroxy-6-(hydroxymethyl)-4oxo-4H-pyran-2-yl]methanes 


\section{Introduction}

Continuously growing interest in convenient and green reaction techniques encourages organic chemists to elaborate new synthetic methodologies. ${ }^{1}$

Tandem reaction is the combination of two or more reactions, which take place in a specific order. ${ }^{2}$ These $^{2}$ reactions are also one-pot processes and hence, they are very powerful method for the efficient construction of complex organic molecules. ${ }^{2}$ These reactions are a part of green chemistry methods, as the stages of isolation and purification of intermediates are omitted, which leads to diminished pollution of the environment. In addition to the intrinsic atom economy and selectivity, the tandem reaction strategy offers significant advantages over conventional linear-type synthesis due to its flexible and convergent nature. ${ }^{3}$

Tandem Knoevenagel-Michael reaction is known in classical organic chemistry, ${ }^{2}$ and until now the investigations in this area are under in progress. ${ }^{4-7}$

The main aim of the modern tandem and cascade strategy is to provide high efficiency and operation simplicity accompanying with low waste formation. This new methodology is based on the pot economy principle and unites it with the atom and step economy strategies. ${ }^{8}$

The organic electrochemistry in the last decades became a new useful method with the important synthetic and ecological advantages. ${ }^{9-12}$ But, the practical usage of electrochemical procedures is often limited with its technical complexity, and usually long processing times. In the course of our study on the electrochemical transformations of organic compounds, we have found the new type of electrochemical transformation, namely the electrocatalytic chain transformation of organic reagents, induced by the catalytic amount of an electrogenerated base in an undivided cell. ${ }^{13}$ We have already successfully applied this electrocatalytic procedure for the synthesis of medicinally relevant 2 -amino-4H-chromene derivatives. ${ }^{14-16}$ This unique electrochemical method utilizes a simple undivided cell and are valuable for the large-scale processes, because of their catalytic nature and the use of a cheap and environmentally responsible chemical reagent electricity. The employment of this electrocatalytic methodology for the initiation of base-activated cascade reactions is very promising, because it provides the combination of the synthetic virtues of the important cascade strategy with the ecological benefits and convenience of the electrocatalytic procedure. ${ }^{17,18}$

In the course of our studies on the tandem, cascade and multicomponent electrochemical transformations of organic compounds ${ }^{19-23}$ we have found a new type of the electrocatalytic process carrying out in undivided cell and have already successfully applied it for some types of the electrochemicaly induced tandem Knoevenagel-Michael reactions of carbonyl compounds and $\mathrm{C}-\mathrm{H}$ acids ${ }^{24-26}$.

The creation of easy and convenient methodology for the selective assembly of biologically active scaffolds in the electrocatalytic multicomponent processes becomes one of the principal goals of the modern electroorganic and green chemistry. ${ }^{27-29}$

Among the different strategies of the drug discovery search, the identification and use of "privileged structures" or scaffolds gained a special attention in the last decades. ${ }^{30}$ Usually, these privileged scaffolds are constructed from heterocyclic ring with specially determined positions of functional groups for target recognition of specified biological receptors. ${ }^{31}$

Heterocycles are the key structural compounds in medicinal chemistry. ${ }^{32}$ They are found in many biologically important molecules such as enzyme, vitamins, natural products and pharmacologically active compounds with antifungal, anti-inflammatory, antibacterial, antioxidant, anticonvulsant, anti-allergic, antiHIV, and anticancer activity. ${ }^{33}$

Among the oxygen containing heterocycles, kojic acid (5-hydroxy-2-hydroxymethyl-4H-pyran-4-one) represents a type of a privileged medicinal scaffold. ${ }^{34}$ Usually, these privileged scaffolds are constructed from 
heterocyclic ring with specially determined positions of functional groups for target recognition of specified biological receptors. ${ }^{35}$ Kojic acid is a known fungal metabolite, which is widely applies in different areas. It was extracted from Aspergillus oryzae, nearly one century ago. ${ }^{36}$ Kojic acid is often used for averting an enzymatic browning in food production and as skin-lightening agent in the cosmetic field. ${ }^{37}$ It showed the potential inhibition of cellular NF-KB activity in human keratinocytes; NF-KB activation is also involved in kojic acid induced anti-melanogenic effect. ${ }^{38}$ It has been also found that kojic acid derivatives show antibacterial, ${ }^{39}$ antiinflammatory, ${ }^{40}$ antiviral, $^{41}$ anti-HIV $^{42}$ and antitumor activity ${ }^{43}$ as well as inhibitors of oxidases, ${ }^{44}$ and tyrosinases. $^{45}$

The introduction of two pharmacology active fragments of kojic acid medicinally privileged scaffold in one molecule, separated by C-aryl-substituted spacer could enhance their pharmacology activity.

We have already implemented some electrochemically induced multicomponent transformations of carbonyl compounds and different $\mathrm{C}-\mathrm{H}$ acids. ${ }^{46,47}$

In continuation of our study we report now the data on the new selective and efficient electrocatalytic multicomponent assembling of aldehydes, and two equivalents of kojic acid into arylbis[3-hydroxy-6(hydroxymethyl)-4-oxo-4H-pyran-2-yl]methanes (Scheme 1).

\section{Results and Discussion}

Electrocatalytic and electrochemically induced cascade reactions are one of the most useful approaches to 'ideal synthesis'. ${ }^{48,49}$

Our present study is dealing with the facile and selective electrochemically induced cascade assembling of aldehydes 1a-m and two equivalents of kojic acid into arylbis[3-hydroxy-6-(hydroxymethyl)-4-oxo-4H-pyran-2yl]methanes 2a-m in alcohols in an undivided cell (Scheme 1, Tables 1, 2).

In the first part of our study, to evaluate the synthetic utility of the electrocatalytic procedure and to investigate the electrolysis conditions, the electrochemically induced cascade assembling of benzaldehyde 1a with two equivalents of kojic acid was carefully studied under conditions of electrolysis in alcohols in an undivided cell (Table 1).

To start this electrochemical research, the electrochemically induced multicomponent reaction of benzaldehyde 1a, and two equivalents of kojic acid in methanol in an undivided cell under constant current conditions was studied (Table 1, entries 1, 2). In this case, when $0.1 \mathrm{~F} / \mathrm{mol}$ of electricity was passed the best yield of 2,2'-[(phenyl)methylene]bis[3-hydroxy-6-(hydroxymethyl)-4H-pyran-4-one] 2a - 43\% - was obtained under conditions with heating up to $63^{\circ} \mathrm{C}$. Under the similar conditions in ethanol and $n$-propanol, 2a was received in 49 and $54 \%$ yields (entries 3 and 4 , Table 1). As it was found in subsequent experiments, the current density $5 \mathrm{~mA} / \mathrm{cm}^{2}\left(I=25 \mathrm{~mA}\right.$, electrodes surface $\left.5 \mathrm{~cm}^{2}\right)$ in an undivided cell, sodium bromide as electrolyte, $n$-propanol as solvent, the temperature near to the boiling point of $n$-propanol and $0.3 \mathrm{~F} / \mathrm{mol}$ of electricity passed, were the optimal conditions for this electrocatalytic chain process of the multicomponent assembling benzaldehyde 1a, and two equivalents of kojic acid. Under these conditions, $2 \mathbf{a}$ was obtained with the highest substance yield $93 \%$ and the current efficiency $310 \%$ (Table 1, entry 7). An increase in the current density up to $10 \mathrm{~mA} / \mathrm{cm}^{2}(I=50 \mathrm{~mA})$ resulted in the decrease of both the substance and the current reaction yields (entry 9, Table 1). A decrease of the current density to $2 \mathrm{~mA} / \mathrm{cm}^{2}(I=10 \mathrm{~mA})$ also led to the decrease of both the current and the substance reaction yields (entry 10, Table 1), more likely due to the insufficient initiation of the electrochemically induced chain reaction in the last case. Thus, the current density $5 \mathrm{~mA} / \mathrm{cm}^{2}$ $\left(I=25 \mathrm{~mA}\right.$, electrodes surface $\left.5 \mathrm{~cm}^{2}\right)$ in an undivided cell, sodium bromide as electrolyte, $n$-propanol as 
solvent, the temperature near to the boiling point of $n$-propanol and $0.3 \mathrm{~F} / \mathrm{mol}$ of electricity passed, were the optimal conditions for the electrochemically induced chain process of the multicomponent assembling benzaldehyde 1a, and two equivalents of kojic acid. Under these conditions, 2,2'-[(phenyl)methylene]bis[3hydroxy-6-(hydroxymethyl)-4H-pyran-4-one] 2 a was obtained with the highest substance yield $93 \%$ and the current efficiency $310 \%$ (Table 1, entry 7 ).<smiles>[R]c1cc(C=O)c([R])c([R])c1[R]</smiles>

$1 a-j$<smiles>O=c1cc(CO)occ1O</smiles><smiles>O=c1cc(CO)occ1O</smiles>

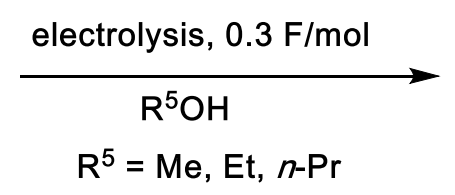

a: $\mathrm{R}^{1}=\mathrm{R}^{2}=\mathrm{R}^{3}=\mathrm{R}^{4}=\mathrm{H}$

b: $R^{1}=R^{2}=R^{4}=H, R^{3}=M e ;$

c: $R^{1}=R^{2}=R^{4}=H, R^{3}=E t ;$

$\mathrm{d}: \mathrm{R}^{1}=\mathrm{R}^{2}=\mathrm{R}^{4}=\mathrm{H}, \mathrm{R}^{3}=t$-Bu;

e: $R^{1}=R^{2}=R^{4}=H, R^{3}=$ OMe;

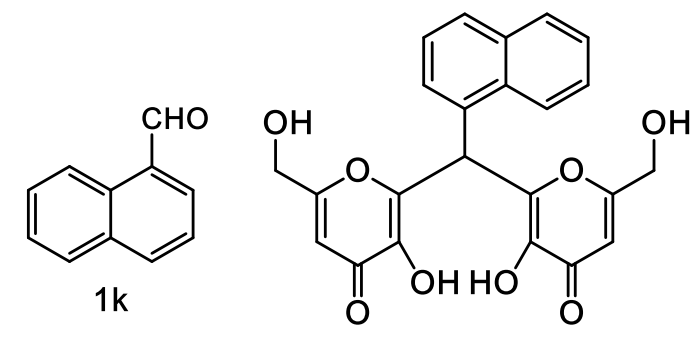

$2 \mathrm{k}$

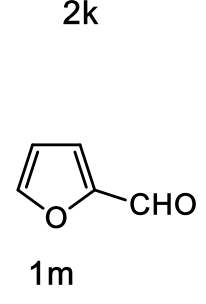<smiles>O=c1cc(CO)oc(C(c2ccco2)c2oc(CO)cc(=O)c2O)c1O</smiles>

$2 m$<smiles>[R]c1cc(C(c2oc(CO)cc(=O)c2O)c2oc(CO)cc(=O)c2O)c([R7])c([R])c1[R]</smiles>

$2 a-j$<smiles>O=Cc1cccnc1</smiles>

11

f: $R^{1}=R^{4}=$ OMe, $R^{2}=R^{3}=H$;

g: $R^{1}=R^{3}=R^{4}=H, R^{2}=F$;

$h: R^{1}=R^{2}=R^{4}=H, R^{3}=F$;

i: $R^{2}=R^{3}=R^{4}=H, R^{1}=C l$;

$\mathrm{j}: \mathrm{R}^{1}=\mathrm{R}^{2}=\mathrm{R}^{4}=\mathrm{H}, \mathrm{R}^{3}=\mathrm{Cl}$.

Scheme 1. Electrocatalytic assembling of aldehydes with two equivalents of kojic acid.

Under the optimal conditions of the electrolysis, aldehydes $1 \mathbf{a}-\mathbf{m}$ and two equivalents of kojic acid in an undivided cell in $n$-propanol were transformed into the arylbis[3-hydroxy-6-(hydroxymethyl)-4-oxo-4H-pyran2-yl]methanes 2 a-m in 71-98\% yields (current efficiency $237-327 \%$ ) (Table 2).

In all these electrochemically induced processes, after the electrolysis was finished, the reaction mixture was concentrated to one fifth of its initial volume and cooled to $0^{\circ} \mathrm{C}$ (ca. $4 \mathrm{~mL}$ ) to crystallize the solid product, which was then filtered out, twice rinsed with an ice-cold ethanol/water solution (4:1, $4 \mathrm{~mL})$, and dried under reduced pressure. 
Table 1. Electrocatalytic assembling of benzaldehyde $1 \mathrm{a}$ and two equivalents of kojic acid ${ }^{a}$

\begin{tabular}{llllllll}
\hline Entry & Solvent & $\begin{array}{l}\text { Temp, } \\
{ }^{\circ} \mathrm{C}\end{array}$ & $I(\mathrm{~mA})$ & $\begin{array}{l}\text { Current } \\
\text { density, } \\
\left(\mathrm{mA} / \mathrm{cm}^{2}\right)\end{array}$ & $\begin{array}{l}\text { Time, } \\
(\mathrm{min})\end{array}$ & $\begin{array}{l}\text { Electricity } \\
\text { passed } \\
(\mathrm{F} / \mathrm{mol})\end{array}$ & $\begin{array}{l}\text { Yield of } \\
\text { 2a (\%) }\end{array}$ \\
\hline 1 & $\mathrm{MeOH}$ & 25 & 25 & 5 & 32 & 0.1 & $17^{\mathrm{b}}(170)$ \\
2 & $\mathrm{MeOH}$ & 63 & 25 & 5 & 32 & 0.1 & $43(430)$ \\
3 & $\mathrm{EtOH}$ & 78 & 25 & 5 & 32 & 0.1 & $49(490)$ \\
4 & $n-\mathrm{PrOH}$ & 97 & 25 & 5 & 32 & 0.1 & $54(540)$ \\
5 & $\mathrm{EtOH}$ & 78 & 25 & 5 & 64 & 0.2 & $63(315)$ \\
6 & $n-\mathrm{PrOH}$ & 97 & 25 & 5 & 64 & 0.2 & $72(360)$ \\
7 & $n-\mathrm{PrOH}$ & 97 & 25 & 5 & 96 & 0.3 & $93(310)$ \\
8 & $n-\mathrm{PrOH}$ & 97 & 50 & 5 & 128 & 0.4 & $88(220)$ \\
9 & $n-\mathrm{PrOH}$ & 97 & 50 & 10 & 48 & 0.3 & $78(260)$ \\
10 & $n-\mathrm{PrOH}$ & 97 & 10 & 2 & 240 & 0.3 & $84(280)$ \\
$11^{\mathrm{c}}$ & $n-\mathrm{PrOH}$ & 97 & 25 & 5 & 96 & 0.3 & $82(273)$ \\
$12^{\mathrm{d}}$ & $n-\mathrm{PrOH}$ & 97 & 25 & 5 & 96 & 0.3 & $85(283)$ \\
\hline
\end{tabular}

Reaction conditions: Benzaldehyde 1a $(5 \mathrm{mmol})$, kojic acid (10 mmol), $\mathrm{NaBr}(1 \mathrm{mmol})$, alcohol (20 mL), iron cathode $\left(5 \mathrm{~cm}^{2}\right)$, graphite anode $\left(5 \mathrm{~cm}^{2}\right)$, undivided cell.

${ }^{a}$ Yield of isolated $\mathbf{2 a}$ (in parentheses, current efficiency, \%). ${ }^{\mathrm{b}}{ }^{1} \mathrm{H}$ NMR data. ${ }^{c} \mathrm{Nal}$ as electrolyte. ${ }^{\mathrm{d}} \mathrm{KBr}$ as electrolyte.

The structure of all new compounds $\mathbf{2 c}, \mathbf{d}, \mathbf{f}-\mathbf{m}$ was confirmed by ${ }^{1} \mathrm{H},{ }^{13} \mathrm{C}$ NMR and IR spectroscopy as well as mass spectrometry and high-resolution mass spectrometry data.

Earlier $\mathbf{2 a}, \mathbf{2} \mathbf{b}, \mathbf{2} \mathbf{j}$ were obtained in $\mathbf{8 0 - 8 8 \%}$ yields by the reaction of 3 equivalents of aldehydes $\mathbf{1 a}, \mathbf{1} \mathbf{b}, \mathbf{1} \mathbf{e}$ (three fold excess of aldehyde) with 2 equivalents of kojic acid in water-methanol mixture in the presence of 1 equivalent of $\mathrm{Na}_{2} \mathrm{CO}_{3}$ as catalyst under microwave irradiation with $400 \mathrm{~W}$ power at $70{ }^{\circ} \mathrm{C} .{ }^{50}$

With the above mentioned results and taking into consideration the mechanistic data on tandem Knoevenagel-Michael reaction, ${ }^{51}$ the following mechanism for the electrocatalytic assembling aldehydes $1 \mathrm{a}-\mathrm{m}$ with two equivalents of kojic acid with the formation of arylbis[3-hydroxy-6-(hydroxymethyl)-4-oxo-4H-pyran2-yl]methanes 2a-m was proposed (Scheme 2).

The first step of this electrochemically induced process is the deprotonation of propanol at the cathode, which leads to the formation of an alkoxide anion. Then, interaction of the alkoxide anion with kojic acid results in the kojic acid anion $\mathbf{A}$ formation (Scheme 2).

The following process in the solution is reaction of the kojic acid anion $\mathbf{A}$ and benzaldehyde $\mathbf{1}$ with the elimination of a hydroxide anion and formation of Knoevenagel adduct $3^{52}$

The hydroxide-anion promoted Michael addition of the next molecule of kojic acid to the electron deficient Knoevenagel adduct $\mathbf{3}$ affords the end product of the electrocatalytic chain process, $\mathbf{2}$ with the regeneration of the alkoxide anion as the last step of the catalytic cycle. Thus, the catalytic chain process continues by the interaction of the alkoxide anion with the next molecule of the kojic acid (Scheme 2). 
Table 2. Electrocatalytic assembling of aldehydes $\mathbf{1} \mathbf{a}-\mathbf{m}$ and two equivalents of kojic acid ${ }^{\text {a }}$

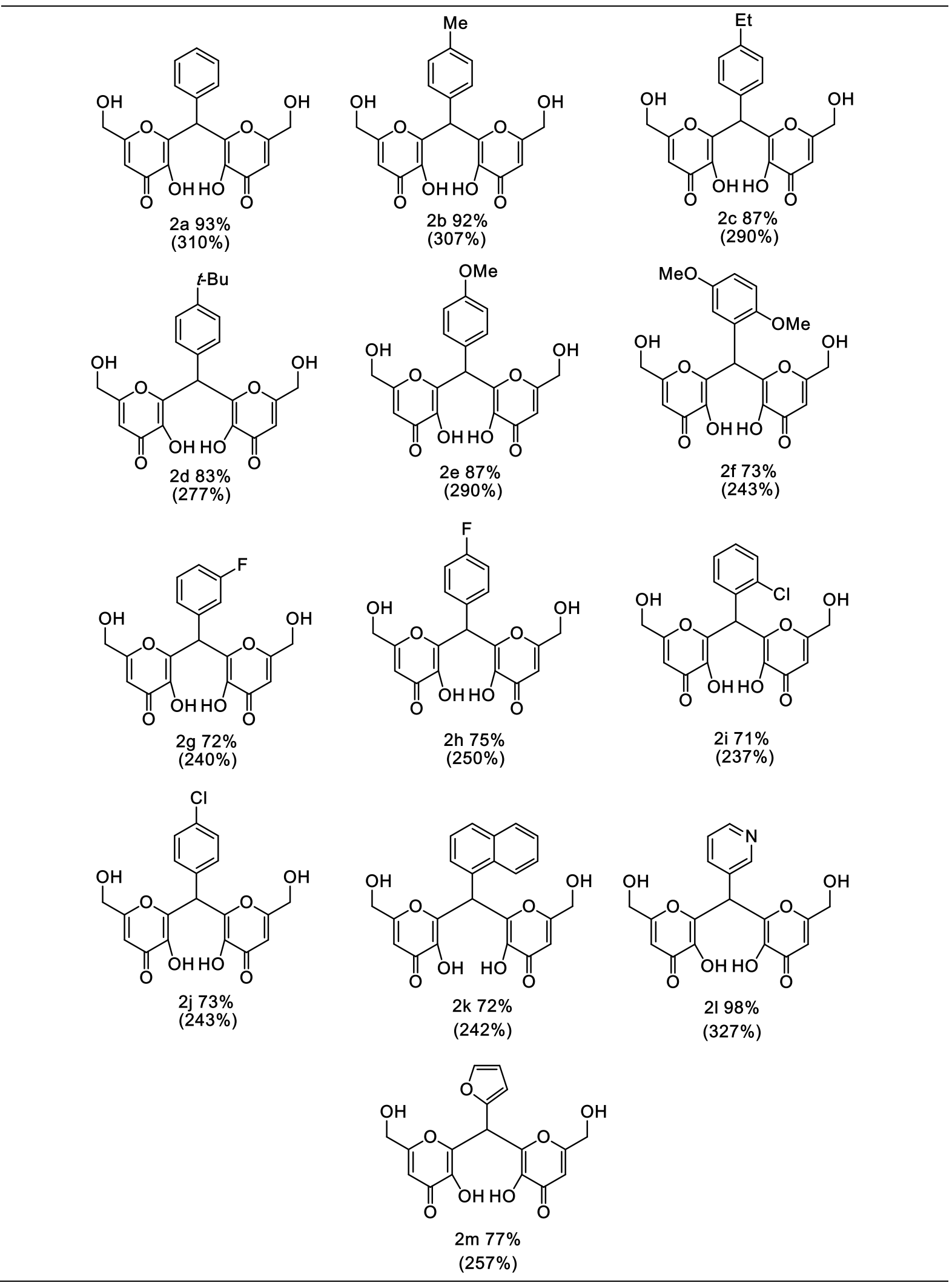

Reaction conditions: Aldehydes $1 \mathrm{a}-\mathrm{k}(5 \mathrm{mmol}), \mathrm{kojic}$ acid $(10 \mathrm{mmol}), \mathrm{NaBr}(1 \mathrm{mmol}), n$-propanol $(20 \mathrm{~mL})$, iron cathode $\left(5 \mathrm{~cm}^{2}\right)$, graphite anode $\left(5 \mathrm{~cm}^{2}\right)$, undivided cell, $97^{\circ} \mathrm{C}, 0.3 \mathrm{~F} / \mathrm{mol}$ of electricity passed.

${ }^{a}$ Yields of isolated $\mathbf{2 a - m}$ (in parentheses, current efficiency). 


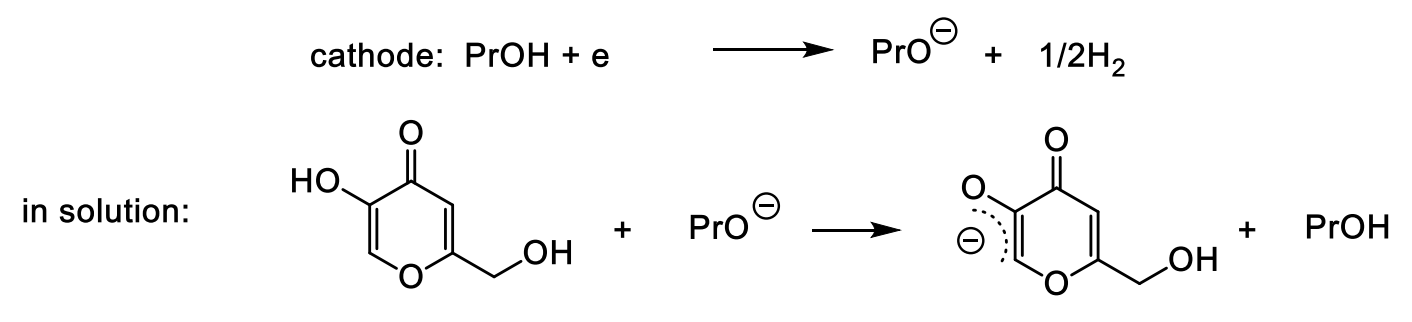

A

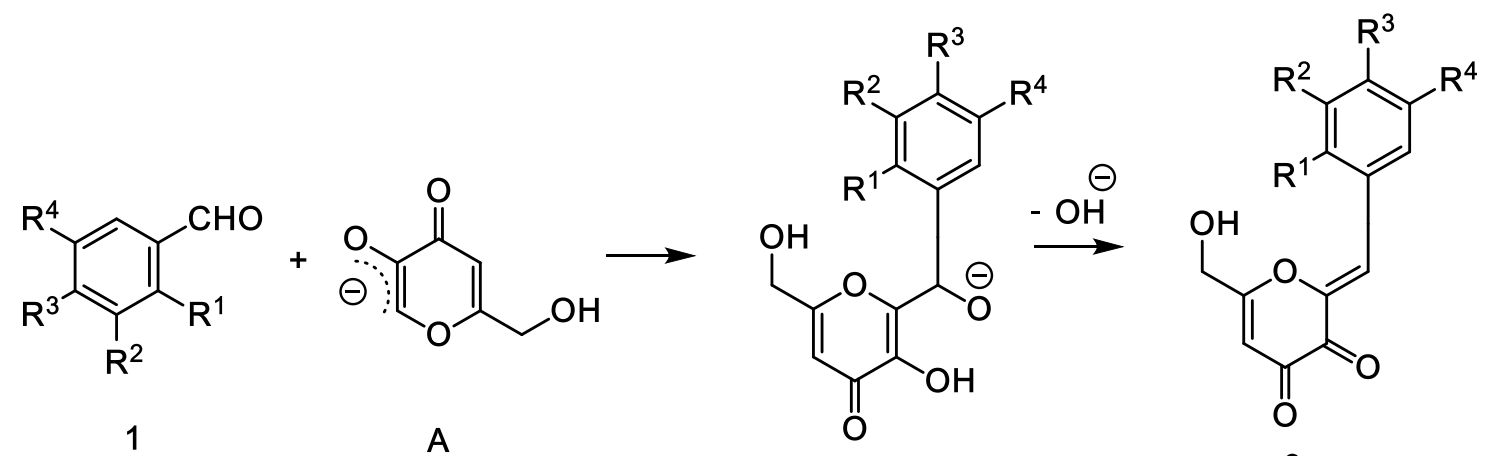

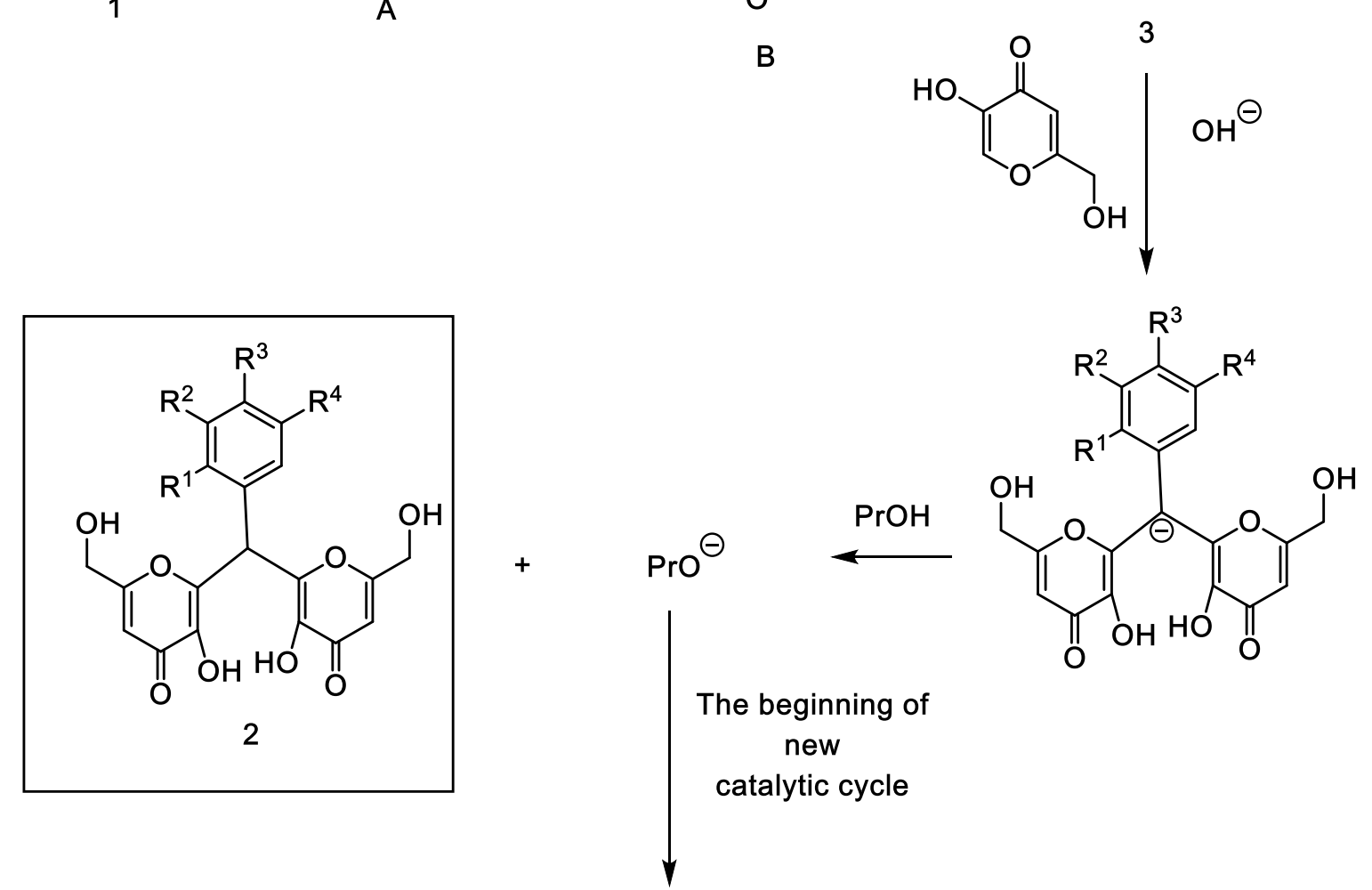

Scheme 2. The mechanism of electrocatalytic transformation of aldehydes with two equivalents of kojic acid.

\section{Conclusions}

The new electrochemically induced fast and highly efficient assembling of aldehydes 1a-m with two equivalents of kojic acid in alcohols in the undivided cell results in the formation of the arylbis[3-hydroxy-6(hydroxymethyl)-4-oxo-4H-pyran-2-yl]methanes $2 \mathrm{a}-\mathrm{m}$ in high $71-98 \%$ yields and with current efficiency 237 $327 \%$. 
This new one-pot electrochemically induced tandem Knoevenagel-Michael process is simple and efficient way to the arylbis[3-hydroxy-6-(hydroxymethyl)-4-oxo-4H-pyran-2-yl]methanes containing two kojic acid fragments separated by $\mathrm{C}$-aryl-substituted spacer, which are promising compounds for different biomedical applications, among them anticonvulsant, anti-AIDS agents and anti-inflammatory remedies.

This electrocatalytic efficient procedure utilizes simple equipment, an undivided cell; available starting compounds, it is easily carried out and the isolation procedure is not complicated. Thus, this new method is valuable from the viewpoint of environmentally benign diversity-oriented large-scale processes.

\section{Experimental Section}

General. All melting points were measured with a Gallenkamp melting-point apparatus and are uncorrected. ${ }^{1} \mathrm{H}$ and ${ }^{13} \mathrm{C}$ NMR spectra were recorded in DMSO- $d_{6}$ with Bruker Avance II 300 spectrometer at ambient temperature. Chemical shift values are relative to $\mathrm{Me}_{4} \mathrm{Si}$. IR spectra were recorded with a Bruker ALPHA-T FTIR spectrometer in $\mathrm{KBr}$ pellets. Mass-spectra $(\mathrm{EI}=70 \mathrm{eV}$ ) were obtained directly with a Kratos MS-30 spectrometer. High-resolution mass spectra (HRMS) (electrospray ionization, ESI) were measured on a BrukermicroTOF II instrument.

General procedure. A solution of aldehyde 1 ( $5 \mathrm{mmol}$ ), kojic acid $(1.42 \mathrm{~g}, 10 \mathrm{mmol}$ ), and sodium bromide $(0.1$ $\mathrm{g}, 1 \mathrm{mmol})$ in $n$-propanol $(20 \mathrm{~mL})$ was electrolyzed in an undivided cell equipped with a magnetic stirrer, a graphite anode and an iron cathode at $97{ }^{\circ} \mathrm{C}$ under a constant current density of $5 \mathrm{~mA} / \mathrm{cm}^{2}(I=25 \mathrm{~mA}$, electrodes square $5 \mathrm{~cm}^{2}$ ) until the catalytic quantity of $0.3 \mathrm{~F} / \mathrm{mol}$ of electricity was passed. After the electrolysis was finished, the reaction mixture was concentrated to one fifth of its initial volume $(c a .4 \mathrm{~mL})$ and cooled to $0^{\circ} \mathrm{C}$ to crystallize the solid compound 2, which was then filtered out, twice rinsed with an ice-cold ethanol/water solution $(4: 1,4 \mathrm{~mL})$, and dried under reduced pressure.

2,2'-[(Phenyl)methylene]bis[3-hydroxy-6-(hydroxymethyl)-4H-pyran-4-one] (2a). White powder; yield $1.73 \mathrm{~g}$, (93\%); mp: $243-245{ }^{\circ} \mathrm{C}$ (decomp.), lit. ${ }^{52} \mathrm{mp}: 244-245.3{ }^{\circ} \mathrm{C}$ (decomp.); ${ }^{1} \mathrm{H}$ NMR (300 MHz, DMSO- $\left.d_{6}\right): \delta 4.24$ (d, 2J $16.3 \mathrm{~Hz}, 2 \mathrm{H}, \mathrm{CH}_{2}$ ), 4.29 (d, ${ }^{2} \mathrm{~J} 16.3 \mathrm{~Hz}, 2 \mathrm{H}, \mathrm{CH}_{2}$ ), 5.63 (br s, 2H, $\left.2 \mathrm{OH}\right), 6.07$ (s, 1H, CH), $6.34(\mathrm{~s}, 2 \mathrm{H}, 2 \mathrm{CH}), 7.29-$ 7.39 (m, 5H, Ph), 9.33 (br s, 2H, $2 \mathrm{OH}) \mathrm{ppm}$.

2,2'-[(4-Methylphenyl)methylene]bis[3-hydroxy-6-(hydroxymethyl)-4H-pyran-4-one] (2b). Yellowish powder; yield $1.78 \mathrm{~g}$, (92\%); mp: $222-223{ }^{\circ} \mathrm{C}$, lit. ${ }^{52} \mathrm{mp}: 223-223.4{ }^{\circ} \mathrm{C} ;{ }^{1} \mathrm{H}$ NMR $\left(300 \mathrm{MHz}, \mathrm{DMSO}-d_{6}\right): \delta 2.27(\mathrm{~s}, 3 \mathrm{H}$, $\left.\mathrm{CH}_{3}\right), 4.22\left(\mathrm{~d},{ }^{2} \mathrm{~J} 16.2 \mathrm{~Hz}, 2 \mathrm{H}, \mathrm{CH}_{2}\right), 4.28$ (d, ${ }^{2} \mathrm{~J} 16.2 \mathrm{~Hz}, 2 \mathrm{H}, \mathrm{CH}_{2}$ ), 5.29-5.90 (br s, 2H, $2 \mathrm{OH}$, exchange with $\left.\mathrm{D}_{2} \mathrm{O}\right)$, $6.01(\mathrm{~s}, 1 \mathrm{H}, \mathrm{CH}), 6.33$ (s, 2H, $2 \mathrm{CH}$ ), 7.15 (d, ${ }^{3} \mathrm{~J} 9.0 \mathrm{~Hz}, 2 \mathrm{H}, 2 \mathrm{CH} \mathrm{Ar}$ ), 7.18 (d, ${ }^{3}$ J 9.0 Hz, 2H, $2 \mathrm{CH} \mathrm{Ar}$ ), 9.06-9.47 (br $\mathrm{s}, 2 \mathrm{H}, 2 \mathrm{OH}) \mathrm{ppm}$.

2,2'-[(4-Ethylphenyl)methylene]bis[3-hydroxy-6-(hydroxymethyl)-4H-pyran-4-one] (2c). Yellowish powder; yield $1.74 \mathrm{~g}$, (87\%); mp: 190-191 ${ }^{\circ} \mathrm{C} ;{ }^{1} \mathrm{H}$ NMR (300 MHz, DMSO-d 6 ): $\delta 1.14\left(\mathrm{t},{ }^{3} \mathrm{~J} 7.6 \mathrm{~Hz}, 3 \mathrm{H}, \mathrm{CH}_{3}\right), 2.56\left(\mathrm{q},{ }^{3} \mathrm{~J} 7.6\right.$ $\mathrm{Hz}, 2 \mathrm{H}, \mathrm{CH}_{2}$ ), 4.23 (d, $\left.{ }^{2} \mathrm{~J} 16.2 \mathrm{~Hz}, 2 \mathrm{H}, \mathrm{CH}_{2}\right), 4.28$ (d, $\left.{ }^{2} \mathrm{~J} 16.2 \mathrm{~Hz}, 2 \mathrm{H}, \mathrm{CH}_{2}\right), 6.00(\mathrm{~s}, 1 \mathrm{H}, \mathrm{CH}), 6.32(\mathrm{~s}, 2 \mathrm{H}, 2 \mathrm{CH}), 7.18$ (d, ${ }^{3}$ J $\left.8.6 \mathrm{~Hz}, 2 \mathrm{H}, 2 \mathrm{CH} \mathrm{Ar}\right), 7.21$ (d, $\left.{ }^{3} \mathrm{~J} 8.6 \mathrm{~Hz}, 2 \mathrm{H}, 2 \mathrm{CH} \mathrm{Ar}\right) \mathrm{ppm} ;{ }^{13} \mathrm{C}$ NMR $\left(75 \mathrm{MHz}, \mathrm{DMSO}-d_{6}\right): \delta 15.5,27.8,40.6$, 59.5 (2C), 109.1, 110.0, 128.3 (4C), 133.6 (2C), 139.3, 142.3, 143.1, 147.0, 167.6, 168.2, 173.7, 174.0 ppm; IR $(\mathrm{KBr}): v 3272,3190,2927,1656,1620,1578,1448,1223,1082,764 \mathrm{~cm}^{-1}$; MS (m/z, relative intensity \%): 400 $\left[\mathrm{M}^{+}\right](14), 258$ (100), 243 (13), 159 (33), 142 (99), 113 (28), 69 (34), 29 (24). MS (ESI): m/z 401.1242[M + H] , calcd for $\mathrm{C}_{21} \mathrm{H}_{20} \mathrm{O}_{8}: 401.1236[\mathrm{M}+\mathrm{H}]^{+}$.

2,2'-[(4-Tert-butylphenyl)methylene]bis[3-hydroxy-6-(hydroxymethyl)-4H-pyran-4-one] (2d). Pale gray powder; yield $1.78 \mathrm{~g}$, (83\%); mp: $211-212{ }^{\circ} \mathrm{C} ;{ }^{1} \mathrm{H}$ NMR (300 MHz, DMSO-d $): \delta 1.24\left(\mathrm{~s}, 9 \mathrm{H}, 3 \mathrm{CH}_{3}\right), 4.24\left(\mathrm{~d},{ }^{2} \mathrm{~J}\right.$ 
$\left.16.4 \mathrm{~Hz}, 2 \mathrm{H}, \mathrm{CH}_{2}\right), 4.29\left(\mathrm{~d},{ }^{2} \mathrm{~J} 16.4 \mathrm{~Hz}, 2 \mathrm{H}, \mathrm{CH}_{2}\right), 6.01(\mathrm{~s}, 1 \mathrm{H}, \mathrm{CH}), 6.32(\mathrm{~s}, 2 \mathrm{H}, 2 \mathrm{CH}), 7.24\left(\mathrm{~d},{ }^{3} \mathrm{~J} 8.3 \mathrm{~Hz}, 2 \mathrm{H}, 2 \mathrm{CH}\right.$ $\mathrm{Ar}), 7.36$ (d, ${ }^{3} \mathrm{~J} 8.3 \mathrm{~Hz}, 2 \mathrm{H}, 2 \mathrm{CH}$ Ar) ppm; ${ }^{13} \mathrm{C}$ NMR (75 MHz, DMSO-d 6 ): $\delta 31.1(3 \mathrm{C}), 34.3,40.3,59.6$ (2C), 109.1 , 110.0, $125.7(2 \mathrm{C}), 128.1(2 \mathrm{C}), 133.4(2 \mathrm{C}), 139.4,142.2,147.0,150.0,167.7(2 \mathrm{C}), 173.7(2 \mathrm{C}) \mathrm{ppm}$; IR (KBr): $v$ $3305,3092,2961,1626,1572,1445,1315,1222,1076,763 \mathrm{~cm}^{-1}$; MS ( $\mathrm{m} / \mathrm{z}$, relative intensity \%): $428\left[\mathrm{M}^{+}\right](16)$, 369 (1), 286 (100), 271 (71), 230 (9), 187 (1), 142 (64), 113 (16), 57 (25), 29 (19). MS (ESI): m/z 429.1551 [M + $\mathrm{H}^{+}$, calcd for $\mathrm{C}_{23} \mathrm{H}_{24} \mathrm{O}_{8}: 429.1549[\mathrm{M}+\mathrm{H}]^{+}$.

2,2'-[(4-Methoxyphenyl)methylene]bis[3-hydroxy-6-(hydroxymethyl)-4H-pyran-4-one] (2e). Yellowish powder; yield $1.75 \mathrm{~g}$, (87\%); mp: $235-237{ }^{\circ} \mathrm{C}$, lit. ${ }^{52} \mathrm{mp}: 235.8-236.7^{\circ} \mathrm{C} ;{ }^{1} \mathrm{H}$ NMR $\left(300 \mathrm{MHz}, \mathrm{DMSO}-d_{6}\right): \delta 3.73(\mathrm{~s}, 3 \mathrm{H}$, $\left.\mathrm{OCH}_{3}\right), 4.17-4.36\left(\mathrm{~m}, 4 \mathrm{H}, 2 \mathrm{CH}_{2}\right), 5.62\left(\mathrm{t},{ }^{3} \mathrm{~J} 5.8 \mathrm{~Hz}, 2 \mathrm{H}, 2 \mathrm{OH}\right), 6.00(\mathrm{~s}, 1 \mathrm{H}, \mathrm{CH}), 6.32(\mathrm{~s}, 2 \mathrm{H}, 2 \mathrm{CH}), 6.91\left(\mathrm{~d},{ }^{3} \mathrm{~J} 8.5\right.$ $\mathrm{Hz}, 2 \mathrm{H}, 2 \mathrm{CH} \mathrm{Ar}), 7.23$ (d, $\left.{ }^{3} \mathrm{~J} 8.5 \mathrm{~Hz}, 2 \mathrm{H}, 2 \mathrm{CH} \mathrm{Ar}\right), 9.28$ (s, 2H, $2 \mathrm{OH}$ ) ppm.

2,2'-[(2,5-Dimethoxyphenyl)methylene]bis[3-hydroxy-6-(hydroxymethyl)-4H-pyran-4-one] (2f). Yellowish powder; yield $1.58 \mathrm{~g}$, (73\%); mp: $221-222{ }^{\circ} \mathrm{C} ;{ }^{1} \mathrm{H} \mathrm{NMR}\left(300 \mathrm{MHz}, \mathrm{DMSO}-d_{6}\right): \delta 3.67\left(\mathrm{~s}, 3 \mathrm{H}, \mathrm{OCH}_{3}\right), 3.68(\mathrm{~s}, 3 \mathrm{H}$, $\left.\mathrm{OCH}_{3}\right), 4.18\left(\mathrm{~d},{ }^{2} \mathrm{~J} 16.1 \mathrm{~Hz}, 2 \mathrm{H}, \mathrm{CH}_{2}\right), 4.23\left(\mathrm{~d},{ }^{2} \mathrm{~J} 16.1 \mathrm{~Hz}, 2 \mathrm{H}, \mathrm{CH}_{2}\right), 6.23(\mathrm{~s}, 1 \mathrm{H}, \mathrm{CH}), 6.31(\mathrm{~s}, 2 \mathrm{H}, 2 \mathrm{CH}), 6.76\left(\mathrm{~d},{ }^{4} \mathrm{~J}\right.$ $2.2 \mathrm{~Hz}, 1 \mathrm{H}, \mathrm{CH} \mathrm{Ar}), 6.85$ (dd, $\left.{ }^{3} \mathrm{~J} 8.9 \mathrm{~Hz},{ }^{4} \mathrm{~J} 2.2 \mathrm{~Hz}, 1 \mathrm{H}, \mathrm{CH} \mathrm{Ar}\right), 6.94$ (d, $\left.{ }^{3} \mathrm{~J} 8.9 \mathrm{~Hz}, 1 \mathrm{H}, \mathrm{CH} \mathrm{Ar}\right) \mathrm{ppm} ;{ }^{13} \mathrm{C} \mathrm{NMR}(75$ $\left.\mathrm{MHz}, \mathrm{DMSO}-d_{6}\right): \delta 36.2,55.4,56.6,59.5(2 \mathrm{C}), 109.2(2 \mathrm{C}), 112.5,112.8,116.0,125.4,142.5,147.0(2 \mathrm{C}), 151.0$, 153.1 (2C), 167.3 (2C), 173.9 (2C) ppm; IR (KBr): v 3283, 3169, 2834, 1617, 1575, 1500, 1452, 1232, 1040, 762 $\mathrm{cm}^{-1}$; MS (m/z, relative intensity \%): $432\left[\mathrm{M}^{+}\right](32), 401$ (2), 292 (60), 290 (100), 259 (25), 191 (24), 142 (98), 137 (34), 69 (41), 29 (51). MS (ESI): $m / z 433.1131[\mathrm{M}+\mathrm{H}]^{+}$, calcd for $\mathrm{C}_{21} \mathrm{H}_{20} \mathrm{O}_{10}: 433.1135[\mathrm{M}+\mathrm{H}]^{+}$.

2,2'-[(3-Fluorophenyl)methylene]bis[3-hydroxy-6-(hydroxymethyl)-4H-pyran-4-one] (2g). Yellowish powder; yield $1.41 \mathrm{~g}$, (72\%); mp: $241-242{ }^{\circ} \mathrm{C}$ (decomp.); ${ }^{1} \mathrm{H}$ NMR (300 MHz, DMSO-d 6 ): $\delta 4.23\left(\mathrm{~d},{ }^{2} J 15.7 \mathrm{~Hz}, 2 \mathrm{H}, \mathrm{CH}_{2}\right)$, $4.29\left(\mathrm{~d},{ }^{2} \mathrm{~J} 15.7 \mathrm{~Hz}, 2 \mathrm{H}, \mathrm{CH}_{2}\right), 5.62(\mathrm{br} \mathrm{s}, 2 \mathrm{H}, 2 \mathrm{OH}), 6.07(\mathrm{~s}, 1 \mathrm{H}, \mathrm{CH}), 6.34(\mathrm{~s}, 2 \mathrm{H}, 2 \mathrm{CH}), 7.07-7.22(\mathrm{~m}, 3 \mathrm{H}, 3 \mathrm{CH}$ Ar), 7.35-7.47 (m, 1H, CH Ar), 9.40 (br s, 2H, $2 \mathrm{OH}$ ) ppm; $\left.{ }^{13} \mathrm{C} \mathrm{NMR} \mathrm{(75} \mathrm{MHz,} \mathrm{DMSO-d}\right): \delta 40.7,59.5$ (2C), 109.2 (2C), $114.7\left(d,{ }^{2} J_{C-F} 22 \mathrm{~Hz}\right), 115.2\left(\mathrm{~d},{ }^{2} J_{C-F} 22 \mathrm{~Hz}\right), 124.6\left(\mathrm{~d},{ }^{4} J_{C-F} 2 \mathrm{~Hz}\right), 130.9\left(\mathrm{~d},{ }^{3} J_{C-F} 8 \mathrm{~Hz}\right), 138.9\left(\mathrm{~d},{ }^{3} J_{C-F} 8 \mathrm{~Hz}\right)$, $142.4(2 \mathrm{C}), 146.2(2 \mathrm{C}), 162.3\left(\mathrm{~d},{ }^{1} J_{C-F} 224 \mathrm{~Hz}\right), 167.8(2 \mathrm{C}), 173.6(2 \mathrm{C}) \mathrm{ppm}$; IR (KBr): $v$ 3412, 3225, 2941, 1624, 1582, 1449, 1234, 1208, 1084, $769 \mathrm{~cm}^{-1}$; MS (m/z, relative intensity \%): $390\left[\mathrm{M}^{+}\right](100), 372$ (7), 331 (48), 303 (33), 261 (46), 250 (36), 142 (26), 109 (7), 69 (58), 29 (48). MS (ESI): m/z 391.0822 [M + H] , calcd for $\mathrm{C}_{19} \mathrm{H}_{15} \mathrm{FO}_{8}: 391.0824[\mathrm{M}+\mathrm{H}]^{+}$.

2,2'-[(4-Fluorophenyl)methylene]bis[3-hydroxy-6-(hydroxymethyl)-4H-pyran-4-one] (2h). Yellowish powder; yield $1.46 \mathrm{~g}$, (75\%); mp: 230-231 ${ }^{\circ} \mathrm{C}$ (decomp.); ${ }^{1} \mathrm{H}$ NMR (300 MHz, DMSO-d $)$ : $\delta 4.23$ (d, ${ }^{2} J 15.8 \mathrm{~Hz}, 2 \mathrm{H}, \mathrm{CH}_{2}$ ), $4.28\left(\mathrm{~d},{ }^{2} \mathrm{~J} 15.8 \mathrm{~Hz}, 2 \mathrm{H}, \mathrm{CH}_{2}\right), 5.94(\mathrm{~s}, 1 \mathrm{H}, \mathrm{CH}), 6.29(\mathrm{~s}, 2 \mathrm{H}, 2 \mathrm{CH}), 7.16\left(\mathrm{dd},{ }^{3} \mathrm{~J} 8.7 \mathrm{~Hz},{ }^{3} \mathrm{JH}_{\mathrm{H} F} 9.9 \mathrm{~Hz}, 2 \mathrm{H}, 2 \mathrm{CH} \mathrm{Ar}\right.$ ), 7.35 (dd, ${ }^{3} \mathrm{~J} 8.7 \mathrm{~Hz},{ }^{4} \mathrm{~J}_{\mathrm{H}-\mathrm{F}} 5.1 \mathrm{~Hz}, 2 \mathrm{H}, 2 \mathrm{CH} \mathrm{Ar}$ ), 8.10-9.25 (br s, 2H, $2 \mathrm{OH}$, exchange with $\left.\mathrm{D}_{2} \mathrm{O}\right) \mathrm{ppm} ;{ }^{13} \mathrm{C} \mathrm{NMR}(75$ MHz, DMSO-d $\left.d_{6}\right): \delta 41.1,59.5(2 \mathrm{C}), 109.2(2 \mathrm{C}), 115.6\left(\mathrm{~d},{ }^{2} J_{C-F} 21 \mathrm{~Hz}, 2 \mathrm{C}\right), 130.4\left(\mathrm{~d},{ }^{3} J_{C-F} 8 \mathrm{~Hz}, 2 \mathrm{C}\right), 132.6\left(\mathrm{~d},{ }^{4} J_{C-F} 2\right.$ $\mathrm{Hz}), 139.4,143.1,146.9(2 \mathrm{C}), 161.5\left(\mathrm{~d},{ }^{1} J_{C-F} 224 \mathrm{~Hz}\right), 167.3(2 \mathrm{C}), 174.3(2 \mathrm{C}) \mathrm{ppm}$; IR (KBr): v 3308, 2902, 1625, $1572,1512,1450,1331,1222,1095,767 \mathrm{~cm}^{-1}$; MS (m/z, relative intensity \%): $390\left[\mathrm{M}^{+}\right]$(2), 261 (2), 248 (34), 191 (1), 149 (44), 142 (16), 109 (71), 69 (40), 29 (100), 18 (50). MS (ESI): m/z 391.0825 [M + H] , calcd for $\mathrm{C}_{19} \mathrm{H}_{15} \mathrm{FO}_{8}: 391.0824[\mathrm{M}+\mathrm{H}]^{+}$.

2,2'-[(2-Chlorophenyl)methylene]bis[3-hydroxy-6-(hydroxymethyl)-4H-pyran-4-one] (2i). Yellowish powder; yield $1.44 \mathrm{~g}$, (71\%); mp: $259-260{ }^{\circ} \mathrm{C}$ (decomp.); ${ }^{1} \mathrm{H}$ NMR (300 MHz, DMSO-d $)$ : $\delta 4.18\left(\mathrm{~d},{ }^{2} J 16.0 \mathrm{~Hz}, 2 \mathrm{H}, \mathrm{CH}_{2}\right)$, $4.23\left(\mathrm{~d},{ }^{2} \mathrm{~J} 16.0 \mathrm{~Hz}, 2 \mathrm{H}, \mathrm{CH}_{2}\right), 5.61$ (br s, $\left.2 \mathrm{H}, 2 \mathrm{OH}\right), 6.30(\mathrm{~s}, 1 \mathrm{H}, \mathrm{CH}), 6.32(\mathrm{~s}, 2 \mathrm{H}, 2 \mathrm{CH}), 7.30-7.41(\mathrm{~m}, 3 \mathrm{H}, 3 \mathrm{CH}$ Ar), 7.44-7.52 (m, 1H, CH Ar), 9.35 (br s, $2 \mathrm{H}, 2 \mathrm{OH}$ ) ppm; ${ }^{13} \mathrm{C} \mathrm{NMR}\left(75 \mathrm{MHz}, \mathrm{DMSO}-d_{6}\right): \delta 39.4,59.4(2 \mathrm{C}), 109.2$ (2C), 127.7, $129.6(2 \mathrm{C}), 130.4,132.9,134.0(2 \mathrm{C}), 142.5,145.7(2 \mathrm{C}), 167.7(2 \mathrm{C}), 173.6(2 \mathrm{C}) \mathrm{ppm}$; IR (KBr): $v 3244$, 2964, 1626, 1575, 1445, 1314, 1214, 1090, 977, $752 \mathrm{~cm}^{-1}$; MS (m/z, relative intensity \%): $408\left[\mathrm{M}^{+}\right]\left({ }^{37} \mathrm{Cl}, 1\right), 406$ $\left[\mathrm{M}^{+}\right]\left({ }^{35} \mathrm{Cl}, 8\right), 319(4), 279\left({ }^{37} \mathrm{Cl}, 10\right), 277\left({ }^{35} \mathrm{Cl}, 21\right), 231\left({ }^{37} \mathrm{Cl}, 44\right), 229\left({ }^{35} \mathrm{Cl}, 68\right), 201(8), 142(14), 101(26), 69$ 
(75), 55 (100), 29 (53). MS (ESI): $m / z 409.0506\left({ }^{37} \mathrm{Cl}\right), 407.0532\left({ }^{35} \mathrm{Cl}\right)[\mathrm{M}+\mathrm{H}]^{+}$, calcd for $\mathrm{C}_{19} \mathrm{H}_{15} \mathrm{ClO}_{8}: 409.0501$ $\left({ }^{37} \mathrm{Cl}\right), 407.0528\left({ }^{35} \mathrm{Cl}\right)[\mathrm{M}+\mathrm{H}]^{+}$.

2,2'-[(4-Chlorophenyl)methylene]bis[3-hydroxy-6-(hydroxymethyl)-4H-pyran-4-one] (2j). Yellowish powder; yield $1.48 \mathrm{~g}$, (73\%); mp: $242-243^{\circ} \mathrm{C}$ (decomp.); ${ }^{1} \mathrm{H}$ NMR (300 MHz, DMSO- $\left.d_{6}\right): \delta 4.23$ (d, ${ }^{2} J 14.8 \mathrm{~Hz}, 2 \mathrm{H}, \mathrm{CH}_{2}$ ), $4.28\left(\mathrm{~d}^{2}{ }^{2} \mathrm{~J} 14.8 \mathrm{~Hz}, 2 \mathrm{H}, \mathrm{CH}_{2}\right.$ ), $5.96(\mathrm{~s}, 1 \mathrm{H}, \mathrm{CH}), 6.31(\mathrm{~s}, 2 \mathrm{H}, 2 \mathrm{CH}), 7.32\left(\mathrm{~d},{ }^{3} \mathrm{~J} 8.4 \mathrm{~Hz}, 2 \mathrm{H}, 2 \mathrm{CH} \mathrm{Ar}\right), 7.40$ (d, ${ }^{3} \mathrm{~J} 8.4$ $\mathrm{Hz}, 2 \mathrm{H}, 2 \mathrm{CH}$ Ar), 8.07-9.49 (br s, $2 \mathrm{H}, 2 \mathrm{OH}$, exchange with $\mathrm{D}_{2} \mathrm{O}$ ) ppm; ${ }^{13} \mathrm{C}$ NMR $\left(75 \mathrm{MHz}\right.$, DMSO- $\left.d_{6}\right): \delta 39.8,59.5$ (2C), $109.2(2 \mathrm{C}), 128.7(2 \mathrm{C}), 130.3(2 \mathrm{C}), 132.2(2 \mathrm{C}), 135.4,143.5,146.7(2 \mathrm{C}), 167.2(2 \mathrm{C}), 174.5(2 \mathrm{C}) \mathrm{ppm}$; IR $(\mathrm{KBr}): v 3330,3086,2939,1620,1572,1451,1332,1214,1093,768 \mathrm{~cm}^{-1}$; MS ( $\mathrm{m} / \mathrm{z}$, relative intensity \%): 268 $\left[\mathrm{M}-\mathrm{C}_{6} \mathrm{H}_{5} \mathrm{O}_{4}+\mathrm{H}\right]+{ }^{+}\left({ }^{37} \mathrm{Cl}, 2\right), 266\left[\mathrm{M}-\mathrm{C}_{6} \mathrm{H}_{5} \mathrm{O}_{4}+\mathrm{H}\right]{ }^{+}\left({ }^{35} \mathrm{Cl}, 8\right), 167\left({ }^{37} \mathrm{Cl}, 4\right), 165\left({ }^{35} \mathrm{Cl}, 15\right), 142(7), 127\left({ }^{37} \mathrm{Cl}, 3\right), 125$ $\left({ }^{35} \mathrm{Cl}, 5\right), 115\left({ }^{37} \mathrm{Cl}, 2\right), 113\left({ }^{35} \mathrm{Cl}, 6\right), 101(6), 69$ (43), 55 (29), 29 (100). MS (ESI): $\mathrm{m} / \mathrm{z} 409.0509\left({ }^{37} \mathrm{Cl}\right), 407.0535$ $\left({ }^{35} \mathrm{Cl}\right)[\mathrm{M}+\mathrm{H}]^{+}$, calcd for $\mathrm{C}_{19} \mathrm{H}_{15} \mathrm{ClO}_{8}: 409.0501\left({ }^{37} \mathrm{Cl}\right), 407.0528\left({ }^{35} \mathrm{Cl}\right)[\mathrm{M}+\mathrm{H}]^{+}$.

2,2'-[(Pyridin-3-yl)methylene]bis[3-hydroxy-6-(hydroxymethyl)-4H-pyran-4-one] (2k). Orange powder; yield $1.96 \mathrm{~g},(98 \%) ; \mathrm{mp:} 157-158{ }^{\circ} \mathrm{C} ;{ }^{1} \mathrm{H}$ NMR $\left(300 \mathrm{MHz}\right.$, DMSO- $\left.d_{6}\right): \delta 4.18-4.36\left(\mathrm{~m}, 4 \mathrm{H}, 2 \mathrm{CH}_{2}\right), 6.04(\mathrm{~s}, 1 \mathrm{H}, \mathrm{CH}), 6.33$ $(\mathrm{s}, 2 \mathrm{H}, 2 \mathrm{CH}), 7.40\left(\mathrm{t},{ }^{3} \mathrm{~J} 7.7 \mathrm{~Hz}, 1 \mathrm{H}, \mathrm{CH} \mathrm{Ar}\right), 7.79\left(\mathrm{~d},{ }^{3} \mathrm{~J} 7.7 \mathrm{~Hz}, 1 \mathrm{H}, \mathrm{CH} \mathrm{Ar}\right), 8.43-8.63(\mathrm{~m}, 2 \mathrm{H}, 2 \mathrm{CH} \mathrm{Ar}), 8.69-10.14$ (br s, $2 \mathrm{H}, 2 \mathrm{OH}$, exchange with $\mathrm{D}_{2} \mathrm{O}$ ) ppm; ${ }^{13} \mathrm{C}$ NMR $\left(75 \mathrm{MHz}\right.$, DMSO- $\left.d_{6}\right): \delta 39.8,59.4(2 \mathrm{C}), 79.2,109.2(2 \mathrm{C})$, 124.0, 132.2, 136.3, 142.4, 145.9, 148.8, 149.2 (2C), 167.7 (2C), 173.6 (2C) ppm; IR (KBr): v 3425, 3234, 1664, $1631,1617,1566,1448,1226,1081,756 \mathrm{~cm}^{-1}$; MS (ESI): $\mathrm{m} / \mathrm{z} 374.0871[\mathrm{M}+\mathrm{H}]^{+}$, calcd for $\mathrm{C}_{18} \mathrm{H}_{15} \mathrm{NO}_{8}: 374.0876$ $[\mathrm{M}+\mathrm{H}]^{+}$.

2,2'-[(1-Naphthyl)methylene]bis[3-hydroxy-6-(hydroxymethyl)-4H-pyran-4-one] (2I). Yellowish powder; yield $1.52 \mathrm{~g},(72 \%) ; \mathrm{mp}: 262-263^{\circ} \mathrm{C}$ (decomp.); ${ }^{1} \mathrm{H}$ NMR $\left(300 \mathrm{MHz}, \mathrm{DMSO}-\mathrm{d}_{6}\right): \delta 4.17\left(\mathrm{~d},{ }^{2} \mathrm{~J} 15.5 \mathrm{~Hz}, 2 \mathrm{H}, \mathrm{CH}_{2}\right), 4.25$ (d, 2J $15.5 \mathrm{~Hz}, 2 \mathrm{H}, \mathrm{CH}_{2}$ ), 5.10-6.18 (br s, 2H, $\left.2 \mathrm{OH}\right), 6.33(\mathrm{~s}, 2 \mathrm{H}, 2 \mathrm{CH}), 6.71(\mathrm{~s}, 1 \mathrm{H}, \mathrm{CH}), 7.38-7.67(\mathrm{~m}, 4 \mathrm{H}, 4 \mathrm{CH} \mathrm{Ar})$, 7.84-8.12 (m, 3H, $3 \mathrm{CH} \mathrm{Ar}$ ), 9.49 (br s, 2H, $2 \mathrm{OH}$ ) ppm; $\left.{ }^{13} \mathrm{C} \mathrm{NMR} \mathrm{(75} \mathrm{MHz,} \mathrm{DMSO-d} d_{6}\right): \delta 37.8,59.5$ (2C), 109.2 (2C), 122.2, 125.8, 126.1, 126.3, 127.0, 128.4, 129.1, 130.9, 132.7, 133.5, 139.3, 142.1, 146.9 (2C), 167.8 (2C), 173.6 (2C) ppm; IR (KBr): v 3454, 3246, 1653, 1632, 1595, 1444, 1245, 1217, 1075, $780 \mathrm{~cm}^{-1}$; MS (ESI): $\mathrm{m} / \mathrm{z}$ $423.1083[\mathrm{M}+\mathrm{H}]^{+}$, calcd for $\mathrm{C}_{23} \mathrm{H}_{18} \mathrm{O}_{8}: 423.1080[\mathrm{M}+\mathrm{H}]^{+}$.

2,2'-[(2-Furyl)methylene]bis[3-hydroxy-6-(hydroxymethyl)-4H-pyran-4-one] $(2 \mathrm{~m})$. Pale brown powder; yield $1.39 \mathrm{~g},(77 \%) ; \mathrm{mp}: 210-211{ }^{\circ} \mathrm{C}$ (decomp.); ${ }^{1} \mathrm{H}$ NMR (300 MHz, DMSO- $\left.d_{6}\right): \delta 4.19-4,33\left(\mathrm{~m}, 4 \mathrm{H}, 2 \mathrm{CH}_{2}\right.$ ), 4.94-5.92 (br s, $2 \mathrm{H}, 2 \mathrm{OH}$, exchange with $\left.\mathrm{D}_{2} \mathrm{O}\right), 6.11(\mathrm{~s}, 2 \mathrm{H}, 2 \mathrm{CH}), 6.28-6.39(\mathrm{~m}, 3 \mathrm{H}, 2 \mathrm{CH}+\mathrm{CH} \mathrm{Ar}), 6.45\left(\mathrm{t},{ }^{3} \mathrm{~J} 2.4 \mathrm{~Hz}, 1 \mathrm{H}\right.$, $\mathrm{CH} \mathrm{Ar}$ ), $7.62\left(\mathrm{~d},{ }^{3} \mathrm{~J} 2.4 \mathrm{~Hz}, 1 \mathrm{H}, \mathrm{CH} \mathrm{Ar}\right), 9.14-9.78$ (br s, $2 \mathrm{H}, 2 \mathrm{OH}$, exchange with $\left.\mathrm{D}_{2} \mathrm{O}\right) \mathrm{ppm} ;{ }^{13} \mathrm{C}$ NMR $(75 \mathrm{MHz}$, DMSO- $\left.d_{6}\right): \delta 35.5,59.5$ (2C), 108.6, $109.2(2 \mathrm{C}), 109.9,110.9,139.3,142.2,142.9,144.7,148.4,167.9(2 \mathrm{C})$, 173.6 (2C) ppm; IR (KBr): v 3364, 3231, 2924, 1647, 1617, 1577, 1450, 1228, 1039, $749 \mathrm{~cm}^{-1}$; MS (ESI): $\mathrm{m} / \mathrm{z}$ $363.0712[\mathrm{M}+\mathrm{H}]^{+}$, calcd for $\mathrm{C}_{17} \mathrm{H}_{14} \mathrm{O}$ : $363.0716[\mathrm{M}+\mathrm{H}]^{+}$.

\section{Acknowledgements}

The reported study was funded by RFBR, project number 19-29-08013.

\section{Supplementary Material}

${ }^{1} \mathrm{H}$ and ${ }^{13} \mathrm{C}$ NMR spectra of all new compounds $\mathbf{2 c , d , f - m}$ are given in the Supplementary Material associated with this paper. 


\section{References}

1. Cioc, R. C.; F. Ruijter, F.; Orru, R. V. A. Green Chem. 2014, 16, 2958-2975. https://doi.org/10.1039/c4gc00013g

2. Ho, T. L. Tandem Organic Reactions, John Wiley \& Sons: New York, 1992.

3. Anastas, P. T.; J. C. Warner, J. C. Green Chemistry: Theory and Practice, Oxford University Press: New York, 2000.

4. Zeynizadeh, B.; Rahmani, S.; Ilkhanizadeh, S. Polyhedron 2019, 168, 48-56. https://doi.org/10.1016/i.poly.2019.04.034

5. Liu, M.; Liu, C-F.; Zhang, J.; Xu, Y-J.; Dong, L. Org. Chem. Front. 2019, 6, 664-668. https://doi.org/10.1039/C8Q001378K

6. Maleki, R.; Koukabi, N.; Kolvari, Appl. Organometal. Chem. 2018, 32, e3905 (1-10). https://doi.org/10.1002/aoc.3905

7. Elinson, M. N.; Sokolova, O. O.; Nasybullin, R. F. Heterocycl. Commun. 2015, 21, 97-101. https://doi.org/10.1515/hc-2015-0046

8. Hayashi, Y. Chem. Sci. 2016, 7, 866-880. https://doi.org/10.1039/C5SC02913A

9. Organic Electrochemistry: Revised and Expand, 5th ed.; Hammerich, O.; Speicer, B. Ed., CRS Press: Boca Raton, 2016.

10. Yan, M.; Kawamata, Y.; Baran, P. S. Angew. Chem. Int. Ed. 2018, 57, 4149-4155. https://doi.org/10.1002/anie.201707574

11. Elinson, M. N.; Makhova, I. V.; Nikishin, G. I. Angew. Chem. Int. Ed. 1988, 77 1716-1717. https://doi.org/10.1002/anie.198817161

12. Ogibin, Y. N.; Elinson, M. N.; Nikishin, G.I. Russ. Chem. Rev. 2009, 78, 89-140. https://doi.org/10.1070/RC2009v078n02ABEH003886

13. For the first report on the chain transformation induced by a catalytic amount of an electrogenerated base in an undivided cell, see: Elinson, M. N.; Feducovich, S. K.; Lizunova T. L.; Nikishin G. I. Tetrahedron 2000, 56, 3063-3069. https://doi.org/10.1016/S0040-4020(00)00195-2

14. Elinson, M. N.; Dorofeev, A. S.; Feducovich, S. K.; S. V. Gorbunov, S. V.; Nasybullin, R. F.; Miloserdov, F. M.; Nikishin, G. I. Eur. J. Org. Chem. 2006, 4335-4339. https://doi.org/10.1002/ejoc.200600544

15. Elinson, M. N.; Dorofeev, A. S.; Ilovaisky, A. I.; Miloserdov F. M.; Feducovich, S. K., Belyakov, P. A.; Nikishin, G. I. Adv. Synth. Cat. 2008, 350, 591-601. https://doi.org/10.1002/adsc.200700493

16. Elinson, M. N.; Vereshchagin, A. N.; Ryzhkov, F. V. Chem. Rec. 2016, 16, 1950-1964. https://doi.org/10.1002/tcr.201600044

17. Ilovaisky, A. I.; Merkulova, V. M.; Elinson, M. N.; Nikishin, G. I. Russ. Chem. Rev. 2012, 81, $381-396$. https://doi.org/10.1070/RC2012v081n05ABEH004244

18. Elinson, M. N.; Vereshchagin, A. N.; Ryzhkov, F. V. Curr. Org. Synth. 2017, 21, $1427-1439$. https://doi.org/10.2174/1385272820666161017170200

19. Nikishin, G. I.; Elinson, M. N.; Lizunova, T. L.; Ugrak, B. I. Tetrahedron Lett. 1991, 32, $2655-2656$. https://doi.org/10.1016/S0040-4039(00)78810-1 
20. Elinson, M. N.; Feducovich, S. K.; Starikova, Z. A.; Vereshchagin, A. N.; Belyakov, P. A.; G. I. Nikishin, G. I.; Mendeleev Commun. 1998, 8, 15-16.

https://doi.org/10.1070/MC1998v008n01ABEH000893

21. Elinson, M. N.; Feducovich, S. K.; Starikova, Z. A.; Vereshchagin, A. N.; Belyakov, P. A.; G. I. Nikishin, G. I.; Tetrahedron 2006, 62, 3989-3996.

https://doi.org/10.1016/j.tetr.2006.02.027

22. Elinson, M. N.; Dorofeev, A. S.; Feducovich, S. K.; Belyakov, P. A.; Nikishin, G. I. Eur. J. Org. Chem. 2007, 3023-3027.

https://doi.org/10.1002/ejoc.200601108

23. E. O. Dorofeeva, M. N. Elinson, A. N. Vereshchagin, N. O. Stepanov, I. S. Bushmarinov, P. A. Belyakov, O. O. Sokolova, G. I. Nikishin, RSC Adv. 2012, 2, 4444-4452.

https://doi.org/10.1039/C2RA20078C

24. Elinson, M. N.;, Merkulova, V. M.; llovaisky, A. I.; Barba, F.; Batanero, B. Electrochim. Acta 2011, 56, 82198223.

https://doi.org/10.1016/electacta.2011.06.059

25. Elinson, M. N.; Merkulova, V. M.; Ilovaisky, A. I.; Demchuk, D. V.; Belyakov, P. A.; G. I. Nikishin, G. I. Mol. Divers. 2010, 14, 833-839.

https://doi.org/10.1021/im00120a002

26. Elinson, M. N.; Merkulova, V. M.; llovaisky, A. I.; Chizhov, A. O.; Barba, F.; Batanero, B. (2014) J. Electrochem. Soc. 2014, 161, G48-G53.

https://doi.org/10.1149/2.104406jes

27. Elinson, M. N.; Dorofeeva, E. O.; Vereshchagin, A. N.; Nikishin G. I. Rus. Chem. Rev. 2015, 84, $485-497$. https://doi.org/10.1070/RCR4465

28. Vereshchagin, A. N.; Elinson, M. N.; Egorov, M. P. RSC Adv. 2015, 5, 98522-98526. https://doi.org/10.1039/c5ra19690f

29. Elinson, M. N.; Gorbunov, S. V.; Vereshchagin, A. N.; Nasybullin, R. F., Goloveshkin, A. S.; Bushmarinov, I. S.; Egorov, M. P. Tetrahedron 2014, 70, 8559-8563.

https://doi.org/10.1016/j.tet.2014.09.066

30. Schneider, P.; Schneider, G. Angew. Chem. Int. Ed. 2017, 56, 7971-7974.

https://doi.org/10.1002/anie.201702816

31. Poupaert, J.; Carato, P.; Colacino, E.; Yous S. Curr. Med. Chem. 2005, 12, 877-885. https://doi.org/10.2174/0929867053507388

32. Arora, P.; V. Arora, V.; Lamba H. S.; Wadhwa, D. Int. J. Pharm. Res. Sci. 2012, 3, 2947-2954. https://doi.org/10.13040/IJPSR.0975-8232.3(9).2947-54

33. Taylor, A. P.; Robinson, R. P.; Fobian, Y. M.; Blamore, D. C.; Jones, L. H.; Fadeyi, O. Org. Biomol. Com. 2016, 14, 6611-6637.

https://doi.org/10.1039/C60B00936K

34. Barreiro E. J. Ch. 1: Privileged scaffolds in medicinal chemistry: an introduction, in Privileged scaffolds in medicinal chemistry: Design, Synthesis, Evaluations, 2015, pp.1-15.

https://doi.org/10.1013/9781782622246-00001

35. Poupaert, J.; Carato, P.; Colacino, E.; Yous, S. Curr. Med. Chem. 2005, 12, $877-885$. https://doi.org/10.2174/0929867053507388

36. Bentley, R. Nat. Prod. Rep. 2006, 23, 1046-1062.

https://doi.org/10.1039/B603758P 
37. Chang,T-S. Int. J. Mol. Sci. 2009, 10, 2440-2475.

https://doi.org/10.3390/ijms10062440

38. Moon, K-Y.; Lee, J.; Ahn K-S.; Kim, Y.; Arch. Pharm. Res. 2001, $24,307-311$. https://doi.org/10.3390/ijms10062440

39. 39. Reddy, B. V. S.; Reddy, M. R.; Madan, C.; Kumar, K. P.; Rao, M. S. Bioorg. Med. Chem. Lett. 2010, 20, 7507-7511.

https://doi.org/10.1016/j.bmcl2010.10.003

40. 40. Rho, H. S.; Ahn, S. M.; Yoo, D, S.; Kim, M. K.; Cho, D. H.; Cho, J. I. Bioorg. Med. Chem. Lett. 2010, 20, 6569-6571.

https://doi.org/10.1016/j.bmcl2010.09.042

41. Aytemir, M. D.; Özçelik, B. Eur. J. Med. Chem. 2010, 45, 4089-4095.

https://doi.org/10.1016/j.ejmech.2010.05.069

42. Tanaka, R.; Tsujii, H.; Yamada, T.; Kajimoto, T.;. Amano, F.; Hasegawa, J.; Hamashima, Y.; Node, M. K.; Katoh, K.; Takebe, Y. Bioorg. Med. Chem. 2009, 17, 5238-5246.

https://doi.org/10.1016/i.bmc2009.05.049

43. You, D. S.; Lee, J.; Choi, S. S.; Rho, H. S.; Cho, D. H. Shin, W. C. Cho, J. Y. Pharmazie 2010, 65, $261-266$. https://doi.org/10.1691/ph.2010.9764

44. Chen, J. S.; Wei C-I.; Roll, R. S.; Otwell, S. W.; Balaban M. D.; Marshall, M. R. J. Agric, Food Chem. 1991, 39, 1396-1401.

https://doi.org/10.1021/j.bmc2009.05.049

45. Cabanes, J.; Chazzara, S.; Carcia-Carmona, F. J. Pharm. Pharmacol. 1994, 46, 982-985.

https://doi.org/10.1111/jf00008a008

46. Elinson, M. N.; Dorofeev, Nasybullin, R. F.; Nikihin, G. I. Synthesis 2008, 1933-1937.

https://doi.org/10.1055/s-2008-1067079

47. Elinson, M. N.; Dorofeev, Nasybullin, R. F.; Fedukovich, S. K.; Nikihin, G. I. Electrochim. Acta 2008, 53, 5033-5038.

https://doi.org/10.1016/electacta.2008.01.066

48. Vereshchagin, A. N.; Elinson, M. N.; Zaimovskaya, T. A.; Nikishin, G. I. Tetrahedron 2008, 64, 9766-9770 https://doi.org/10.1016/j.tet.2008.060

49. P. A. Wender, Nat Prod Rep 31 (2014), 433-440.

https://doi.org/10.1039/C4NP00013G

50. Hua, P-P.; Zhub, C-F.; Zhoa, T. J. Chem. Res. 2012, 36, 450-452.

https://doi.org/10.3184/174751912X13383100102119

51. Elinson, M. N.; Nasybullin, R. F.; Nikihin, G. I. J. Electrochem. Soc. 2013, 160, G3053-G3057. https://doi.org/10.1149/2.009307jes

52. Patai S., Y. Israeli, Y. J Chem Soc (1960), 2025-2030.

http://dx.doi.org/10.1039/jr9600002025

This paper is an open access article distributed under the terms of the Creative Commons Attribution (CC BY) license (http://creativecommons.org/licenses/by/4.0/) 